\title{
Publicación científica en especialidades de Neurología y Psiquiatría en el Perú a través del SCImago Journal and Country Rank.
}

Scientific publication in the field of Neurology and Psychiatry in Peru; through the SCImago Journal and Country Rank.

Joel I. Atencio-Paulino, ${ }^{1, b}$, Waldir Paucar-Huaman ${ }^{2, a}$, Ivan R. Condor-Elizarbe. ${ }^{1, a}$

\section{Estimados Editores:}

En el Perú, el Dr. Julio Oscar Trelles y el Dr. Honorio Delgado, en el campo de la neurología y psiquiatría, respectivamente, fueron pioneros en la investigación y condujeron el desarrollo de dichas especialidades a un nivel importante respecto a Latinoamérica (1). Además en la investigación neuropsiquiátrica, nuestro país siempre aportó trabajos desde la época colonial donde se mostraba la admiración por las técnicas neuroquirúrgicas antiguas, hasta patologías únicas como fue la Enfermedad de Monge o "Enfermedad de los Andes" donde el Dr. Carlos Monge describe los trastornos nerviosos en la altura, publicando sus hallazgos en 1939 en la "Revista de Neuropsiquiatría" e internacionalmente en 1943 en la Physiological Reviews, refiriéndose a la "Chronic Mountain Sickness" (2). Asimismo, la investigación psiquiátrica tiene dos hitos importantes que son la publicación científica a través de la revista más antigua en lengua española como es la Revista de Neuropsiquiatría con una aparición ininterrumpida, y la creación del Instituto Nacional de Salud Mental "Honorio Delgado-Hideyo Noguchi” (3).

Los parámetros bibliométricos para ver el impacto de las publicaciones científicas no son uniformes y aunque se llega a consensos, siempre podrá haber críticas respecto a cuáles son los mejores. La base de datos SCOPUS se creó como competencia a la tradicional Web of Science (WoS) de la plataforma Web of Knowledge para determinar el impacto de la publicación científica en el mundo, sus ventajas incluyen tener el $100 \%$ de indexación de las bases de datos MEDLINE, EMBASE y COMPEDEX y un acceso más libre, siendo su factor impacto (SJR) parte importante para la calificación del ranking de universidades según el "Webometrics" (Ranking Web of World Universities) (4-6). No existe un ganador respecto a cuál es mejor si SCOPUS o WoS, pero sí existe concordancia entre estas dos bases de datos. El SCImago Journal and Country Rank, fundado por el Dr. Félix de Moya, es un portal de evaluación de revistas y de países basado en la información que dispone y almacena la base de datos SCOPUS (4-6).

Para fines de este artículo se extrajo del portal SCImago Journal and Country Rank (SJR), información bibliométrica en la categoría: "All subject areas" y en la subcategoría: "Neurology (clinical)" y luego "Psychiatry and Mental Health" (7), obteniéndose: 1) el número total de documentos en los que al menos uno de los autores tiene afiliación correspondiente al Perú, considerando todos los tipos de documentos, incluidos los citables y no citables; 2) el índice h que expresa el número de artículos (h) que han recibido al

Facultad de Medicina Humana, Universidad Nacional del Centro del Perú. Junin, Perú.

2 Red de Salud de Pangoa-Junín. Junin, Perú.

a Médico-Cirujano, ${ }^{\mathrm{b}}$ Estudiante de Medicina. 
menos h citas; 3) el número de citas realizadas a los artículos publicados y 4) el promedio de citas por documento.

La publicación respecto a otros países de Latinoamérica en el campo de la neurología y psiquiatría es baja. Aunque durante los últimos años se ha venido acrecentando tal como se aprecia en los gráficos 1 y 2 , esto no es suficiente; es así que entre los años 1996-2018 se estima que se publicaron unos 337 artículos en la subcategoría Neurology (clinical) con un índice $\mathrm{h}=35$, ocupando el octavo lugar de 42 países registrados para Latinoamérica, y unos 260 artículos con un índice $\mathrm{h}=29$ en la subcategoría Psychiatry and Mental Health (7).
En nuestro país las siguientes instituciones y asociaciones: Sociedad Peruana de Neurología, Instituto Nacional de Ciencias Neurológicas, Asociación Psiquiátrica Peruana, Instituto Nacional de Salud Mental "Honorio delgado-Hideyo Noguchi", hospitales de complejidad importante de EsSalud de atención neuropsiquiátrica, filiales de dichas instituciones en ciudades como Arequipa y Trujillo, entre otras más asociaciones privadas sin fines de lucro, son vitales e importantes, para reforzar no solo la capacidad asistencial sino también la capacidad investigadora en todo el Perú, recordando el motor y uno de los pilares de los fundadores de la "Revista de Neuropsiquiatría": el trabajo intersectorial entre dichas especialidades.

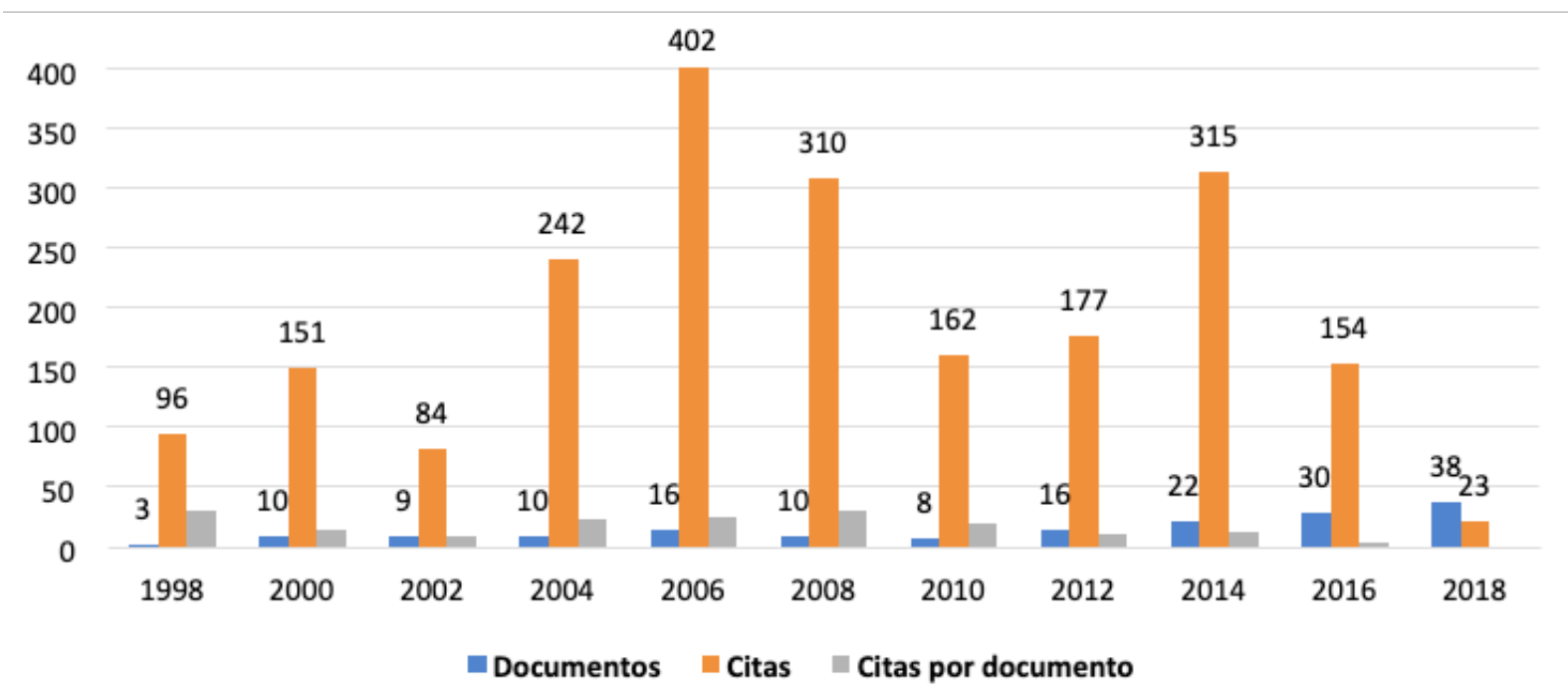

Gráfico 1. Producción de investigación en la subcategoría Neurology (clinical) en el Perú, 1998-2018 (número de artículos). Fuente: Scimago Journal and Country Rank.

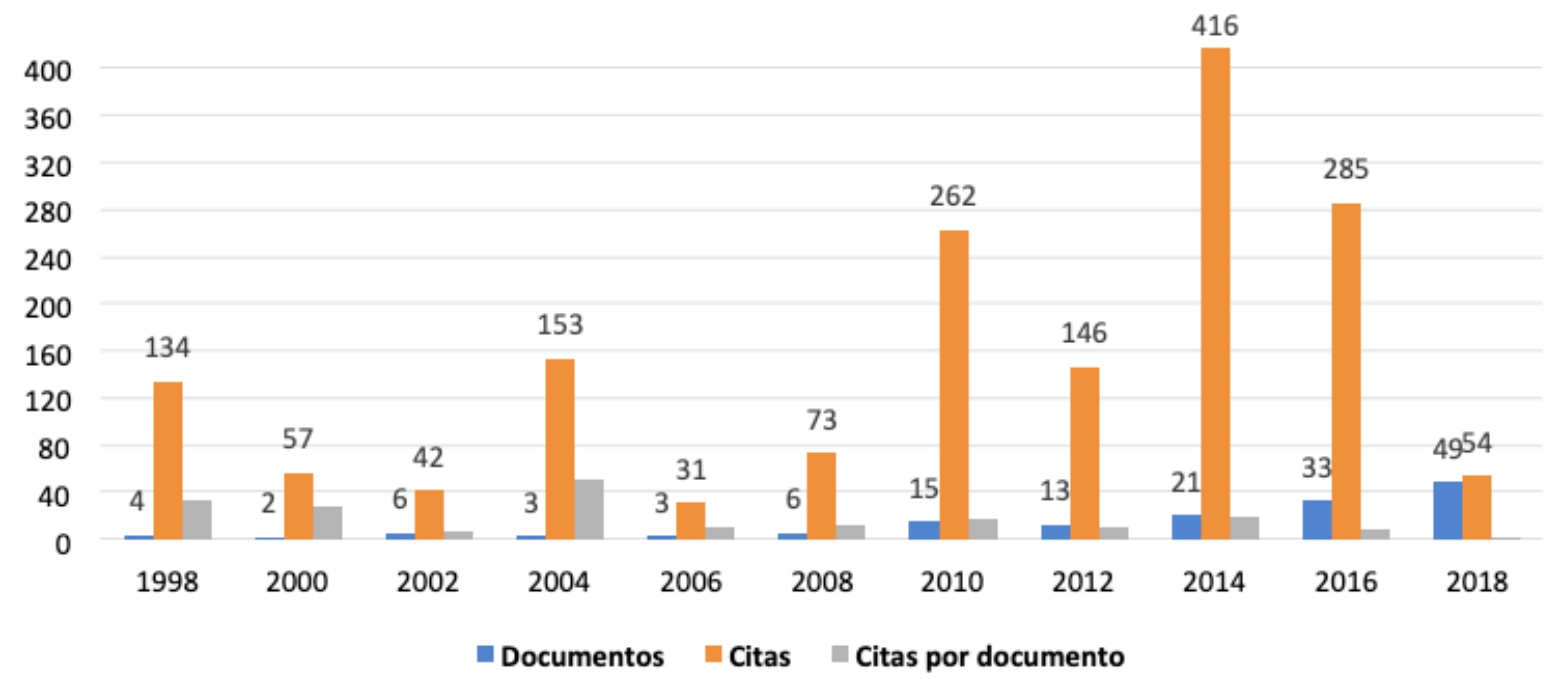

Gráfico 2. Producción de investigación en la subcategoría Psychiatry and Mental Health en el Perú, 1998-2018 (número de artículos). Fuente: Scimago Journal and Country Rank. 
Publicación científica en especialidades de Neurología y Psiquiatría en el Perú a través del SCImago Journal.

\section{Correspondencia:}

Joel Atencio.

Jr. Zorritos 1399, Block 42. Dept. 403, Cercado de Lima; código postal: 5112006

Correo electrónico: yoel_005@yahoo.com

\section{REFERENCIAS BIBLIOGRÁFICAS.}

1. Alva V. La Revista de Neuro-Psiquiatría: reflexiones sobre sus fundadores, sus «laboratorios» y su historia institucional. Rev Neuro-Psiquiatr. 2015; 78(2):102-9.

2. Alzamora-Valdez E. Aportes para la historia de la neurología en el Perú. An Fac Med. 1944; 27(2):96-138. 3. Mariátegui J. La Psiquiatría Peruana: presente y futuro. Rev Neuropsiquiatr. 1999; 62(1):3-13.
4. Granda-Orive J, Alonso-Arroyo A, García-Río F, Solano-Reina S, Jiménez-Ruiz C, Aleixandre-Benavent R. Ciertas ventajas de Scopus sobre Web of Science en un análisis bibliométrico sobre tabaquismo. Rev Esp Doc Científica. 2013; 36(2):011.

5. Zacca-González G, Chinchilla-Rodríguez Z, Vargas-Quesada B, de Moya-Anegón F. Patrones de comunicación e impacto de la producción científica cubana en salud pública. Rev Cuba Salud Pública. 2015; 41(2):200-16.

6. Merlino-Santesteban C. Desempeño científico argentino en cinco áreas prioritarias de I+D. Una mirada a través del SCImago Journal \& Country Rank. Rev Iberoam Cienc Tecnol Soc - CTS. 2013; 8(22):33-56.

7. Scimago. Scimago Journal \& Country Rank. Madrid: Scimago; 2019. (Fecha de acceso setiembre del 2019) Disponible en: https://www.scimagojr.com/journalrank. php 